Mathematisches Forschungsinstitut Oberwolfach

Report No. 11/2011

DOI: $10.4171 /$ OWR/2011/11

\title{
Mini-Workshop: The Homotopy Interpretation of Constructive Type Theory
}

\author{
Organised by \\ Steve Awodey, Pittsburgh \\ Richard Garner, Sydney \\ Per Martin-Löf, Stockholm \\ Vladimir Voevodsky, Princeton
}

February 27th - March 5th, 2011

\begin{abstract}
Over the past few years it has become apparent that there is a surprising and deep connection between constructive logic and higherdimensional structures in algebraic topology and category theory, in the form of an interpretation of the dependent type theory of Per Martin-Löf into classical homotopy theory. The interpretation results in a bridge between the worlds of constructive and classical mathematics which promises to shed new light on both. This mini-workshop brought together researchers in logic, topology, and cognate fields in order to explore both theoretical and practical ramifications of this discovery.
\end{abstract}

Mathematics Subject Classification (2000): 03B15, 55Pxx.

\section{Introduction by the Organisers}

The mini-workshop The Homotopy Interpretation of Constructive Type Theory, organised by Steve Awodey (Pittsburgh), Richard Garner (Sydney), Per MartinLöf (Stockholm) and Vladimir Voevodsky (Princeton) was held between February 27th and March 5th 2011. It brought together researchers from three different continents and a number of different fields; some known for their work in constructive mathematics and categorical logic, others for their work in higher-dimensional category theory, and others still for their work in algebraic topology and homotopy theory. What brought this seemingly disparate group of individuals together was a desire to understand more completely a connection recently established between these three areas: one mediated by the dependent type theory of Per Martin-Löf. 
Martin-Löf developed his dependent type theory in the 1970's to provide a rigourous constructive foundation for mathematics. Besides its philosophical and theoretical interest, constructive type theory is of increasing practical importance, since its constructivity is also a kind of computability: thus it can be viewed as a particularly sophisticated kind of programming language, and in this guise, it provides the foundation for a number of computerised "proof assistants" - such as Epigram, Agda and Coq - which permit the large-scale formalisation and verification of parts of mathematics and computer science.

As with any logical system, it is meaningful to ask what an appropriate notion of model might be for dependent type theory. It is in considering this question that the connection with homotopy theory and higher category theory is manifested. Since the 1980's we have had a notion of categorical model for dependent type theory: this being a category equipped with certain extra structure suitable for modelling the entities present in the logic. But what has only been realised over the past five or so years is that the extra categorical structure required to model the notion of equality in dependent type theory corresponds in a very precise manner to the structure of a weak factorisation system such as occurs in Quillen's theory of abstract homotopical algebra, and which is of importance in the study of higher-dimensional category theory.

This basic insight suggests that further, precise, links might be made between the three areas mentioned above, and a small but growing body of work has begun to show that this is the case. The purpose of this workshop was to bring together researchers active in these areas - and interested in their intersection - in order to take stock of the current situation and to determine in which further directions these ideas might fruitfully be pushed. A particular goal of the workshop was to provide a forum for Vladimir Voevodsky - who has been considering questions in this area since 2006, but working broadly independently of other involved researchers - to communicate at length his own ideas about, and visions for, the topic at hand.

In line with this latter objective, approximately half of the itinerary for the workshop was given over to a series of lectures by Voevodsky. In these, he described his programme of "univalent foundations"; its objective being to exploit the connection between dependent type theory and homotopy theory to provide a foundation for mathematics, based on dependent type theory, which is intrinsically homotopical - in the sense of having as its basic entities not only sets, but the entire taxonomy of homotopy types. This foundational endeavour is not merely of theoretical interest; for as described above, Martin-Löf's type theory underpins a number of computer-based proof assistants, and an important part of the univalent-foundational project is that its toolset should include a workable computer system in which modern mathematics, including its homotopy-theoretic and higher-dimensional aspects, may be formalised. These two sides of the projectthe theoretical and the practical - were reflected in two streams to Voevodsky's lectures. The first dealt with the practical issues surrounding the development of 
mathematics within these foundations, aided by the proof-assistant Coq. The second was devoted to showing the consistency of the univalent foundations relative to classical mathematics; it did so by exhibiting a model for these foundations - which are an extension of Martin-Löf's dependent type theory by a homotopy-theoretic reflection principle called the "univalence axiom" - in the algebraic topologist's category of simplicial sets.

The remaining half of the workshop's itinerary was filled by talks from its other participants. Given the rather diverse backgrounds of the audience, it was thought prudent to devote the first two days of these talks to expository surveys which, taken together, would ensure that everyone present was fully equipped to process the material that was being presented to them. The remainder of the talks described some of the most recent developments in the area, both in relation to Voevodsky's univalent foundations, and otherwise. The programme was rounded out by an open problems session which helped to crystallise those questions around which future research might accrete.

The organisers would like to advance their thanks, on behalf of all the workshop participants, to Mathematisches Forschungsinstitut Oberwolfach for their efficient and unobtrusive arrangement of the practical aspects of this workshop, and for providing a quietly inspiring backdrop to a most scientifically fruitful meeting. 



\section{Mini-Workshop: The Homotopy Interpretation of Constructive Type Theory}

\section{Table of Contents}

Vladimir Voevodsky

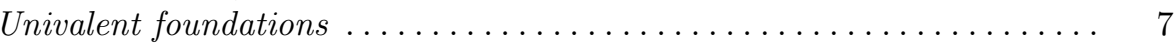

Andrej Bauer

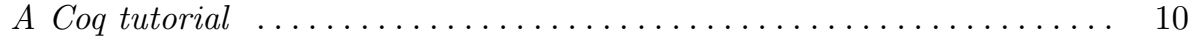

Thomas Streicher

Introduction to Type Theory and its Semantics .............. 11

Michael Shulman

Introduction to Homotopy Theory .................... 13

Richard Garner

Homotopical aspects of type theory ..................... 14

Thierry Coquand

Equality and dependent type theory $\ldots \ldots \ldots \ldots \ldots \ldots \ldots \ldots \ldots \ldots \ldots$

Nicola Gambino

The Univalence Axiom and Function Extensionality ............. 17

Peter Aczel

Logic-enriched type theory $\ldots \ldots \ldots \ldots \ldots \ldots \ldots \ldots \ldots \ldots \ldots \ldots \ldots$

André Joyal

Remarks on homotopical logic ...................... 19

Michael A. Warren (joint with Pieter Hofstra)

A combinatorial realizability model of 1-truncated type theory . . . . . . 22

Benno van den Berg (joint with Richard Garner)

Homotopy-theoretic models of type theory ................ 25

Open problems session $\ldots \ldots \ldots \ldots \ldots \ldots \ldots \ldots \ldots \ldots \ldots \ldots \ldots \ldots \ldots$ 



\section{Abstracts \\ Univalent foundations \\ VLADIMIR VOEVODSKY}

This series of lectures was an introduction to "univalent foundations" - a new foundational system for mathematics based on type theory and homotopy theorytouching on their overall philosophy, some of the challenges involved in developing mathematics in these foundations, and the question of their consistency relative to the standard foundations.

The broad motivation behind univalent foundations is a desire to have a system in which mathematics can be formalised in a manner which is as natural as possible. Whilst it is possible to encode all of mathematics into Zermelo-Fraenkel set theory, the manner in which this is done is frequently ugly; worse, when one does so, there remain many statements of ZF which are mathematically meaningless. This problem becomes particularly pressing in attempting a computer formalisation of mathematics; in the standard foundations, to write down in full even the most basic definitions - of isomorphism between sets, or of group structure on a setrequires many pages of symbols. Univalent foundations seeks to improve on this situation by providing a system, based on Martin-Löf's dependent type theory, whose syntax is tightly wedded to the intended semantical interpretation in the world of everyday mathematics. In particular, it allows the direct formalisation of the world of homotopy types; indeed, these are the basic entities dealt with by the system.

The lectures given were divided into two groups. The first dealt with the practical challenges of developing and formalising mathematics within this foundational system, aided by the mechanised proof-assistant Coq. The second was devoted to showing the consistency of these foundations relative to the usual ones; this was done by constructing a model of the univalent foundations in the category of simplicial sets.

The first group of lectures were given largely with reference to a Coq "proof script" - a text file providing commands to be executed by the Coq proof-assistant. These commands allow one to make definitions and to prove lemmas and theorems concerning those definitions, as one is accustomed to do in mathematics, all within an ambient logic which is (an extension of) Martin-Löf's dependent type theory. Crucially, it is possible - by virtue of the homotopy-theoretic aspects of this type theory - to adopt the position that the types which make up the Coq system should have their intended interpretation as topological spaces. By making definitions which reflect this position, one can in fact develop quite substantial parts of homotopy theory in this framework. We now summarise some key points in such a development.

In keeping with the intended interpretation, we shall denote the identity types of Martin-Löf type theory not by $\operatorname{ld}_{A}(a, b)$ but rather by paths ${ }_{A}(a, b)$, thinking of this as the space of paths from $a$ to $b$ in the space $A$. This is reasonable, since all 
of the operations we expect a path space to support- such as the composability and reversibility of paths - are derivable in the type theory.

Now to any type $A$, we associate a new type iscontr $A$, defined as $\Sigma x: A$. Пy: $A$. paths $A x y$. Under the intended interpretation, an element of this type is given by a point $x$ in the space $A$ and a family of paths from this $x$ to each point $y \in A$, varying continuously in the parameter $y$. Such an element exists just when the space $A$ is contractible in the usual sense.

The notion of contractibility sits at the bottom of a hierarchy of notions that may be attached to a type. We define a type $A$ to be of $h$-level 0 if it is contractible, and of $h$-level $n+1$ if, inductively, for all $x, y: A$, paths $A(x, y)$ is of $h$-level $n$. We say that a type is a property if of $h$-level 1 , and that it is a set if of $h$-level 2. All of these notions may be formalised as new types within our theory; so, for instance, we define isaprop $A$ to be the type $\Pi x, y: A$. iscontr( paths $\left._{A}(x, y)\right)$. From these definitions, it is possible to prove, for instance, that for any type $A$ we have isaprop $(\operatorname{iscontr}(A))$, and isaprop(isaprop $(A))$, and more generally that isaprop(ishlevel $(n, A))$.

The next important type-theoretic definition is that of weak equivalence. We first define the homotopy fiber of a map $f: A \rightarrow B$ over a point $b: B$ to be the type hfiber $f b:=\Sigma x: A$. paths $_{B}(f x, b)$. We now define a map $f$ to be a weak equivalence if all of its homotopy fibers are contractible; formally, we define isweq $f$ to be the type $\Pi x: A$. iscontr(hfiber $f x$ ), and define the type weq $A B$ of weak equivalences from $A$ to $B$ to be $\Sigma f: A \rightarrow B$. isweq $f$. We may now prove, for instance, that a map $f: A \rightarrow B$ is a weak equivalence if and only if there exists a map $g: B \rightarrow A$ and homotopies $\Pi x: A$. paths $A(x, g f x)$ and $\Pi y: B$. paths $B(f g y, y)$.

We may now go on to develop basic properties of weak equivalences, and of their interaction with the type-theoretic constructors, and of their interaction with the hierarchy of $h$-levels. It is also possible to develop quite directly further aspects of homotopy theory, such as the theory of homotopy fiber sequences. However, let us close this discussion of the first group of lectures by introducing the univalence axiom; this is the one major addition to Martin-Löf's type theory required to make its behaviour sufficiently close to that of the intended interpretation.

For this we suppose provided with a type-theoretic universe $U$; this corresponds to something like an inaccessible cardinal in the Zermelo-Fraenkel foundations. Now for any $A, B: U$, it is possible to construct a term $\theta:$ paths $_{U}(A, B) \rightarrow$ weq $A B$, showing that if two types in the universe have a path between them, then those types are weakly equivalent. The univalence axiom states that the map $\theta$ should itself be a weak equivalence for every $A, B: U$; in other words, that the homotopy theory of the types in the universe should be fully and faithfully reflected by the equality on the universe. An important consequence of this axiom is the principle of function extensionality; that if two elements $f, g: \Pi x: A . B(x)$ are pointwise homotopic, in the sense that $\Pi x: A$. paths ${ }_{B(x)}(f x, g x)$ is inhabited, then they are joined by a path in the function space; that is, an element of paths $_{\Pi x: A . B(x)}(f, g)$. 
This concludes our discussion of the first group of lectures, and we now move on to the second, which was devoted to proving the consistency of the univalent foundations relative to the standard ones. These began by describing an appropriate mathematical representation of the syntax of a type theory. The basis of such a representation is a general system of expressions which is, by definition, a finitary (= filtered-colimit preserving) monad $T$ on the category of sets. The value of such a monad at the $n$-element set is to be interpreted as the set of raw syntactic expressions with $n$ free variables; we typically generate such monads as submonads of free monads on a set of constructors. Given such a $T$, a type theory over $T$ is given by a pair of predicates determining those lists of raw syntactic expressions which represent valid judgements of the type theory at hand.

This gives a robust mathematical representation of the syntax of a type theory; but in practice it is more convenient to represent such type theories as structured categories. For this we essentially use Cartmell's notion of contextual category: this being a category $\mathcal{C}$ - to be thought of as the category whose objects are contexts in the given type theory and whose morphisms are substitutions - equipped with certain extra structure allowing this intuition to be realised. For example, the objects of $\mathcal{C}$ are graded by $\mathbb{N}$ - the degree of an object describing the length of the context it represents - such that there is a unique object of degree 0 , which is terminal, and such that any object $X$ of non-zero degree comes equipped with a specified map $p_{X}: X \rightarrow \mathrm{ft} X$, which represents the projecting-away of the last component of the context. We do not rehearse the remaining details here.

We may now go on to construct from the category of simplicial sets a contextual category which supports the interpretation of all the constructors of Martin-Löf's dependent type theory, plus the univalence axiom; this then proves the relative consistency of the univalent foundations. The key to the construction is as follows. We define a universe in a category $\mathcal{C}$ to be a map $p: \tilde{U} \rightarrow U$ together with a chosen pullback of that map along every $f: X \rightarrow U$. Assuming $\mathcal{C}$ to have a terminal object, we may define from this a contextual category $\mathbb{C}(\mathcal{C}, p)$ as follows. There is (necessarily) a unique object of degree 0 ; the objects of degree 1 are maps $f: 1 \rightarrow U$ in $\mathcal{C}$; the objects of degree 2 are pairs of a map $f: 1 \rightarrow U$ together with a map $g: f^{*}(\tilde{U}) \rightarrow U$; objects of degree 3 are triples of $f: 1 \rightarrow U, g: f^{*}(\tilde{U}) \rightarrow U$ and $h: g^{*}(\tilde{U}) \rightarrow U$; and so on.

If $\mathcal{C}$ is locally cartesian closed, it is possible to define what it means, for instance, for the universe $p: \tilde{U} \rightarrow U$ to be closed under dependent products. For this, let $q: \tilde{V} \rightarrow V$ denote the "universal dependent family in $U$ "; it is the image of $p: \tilde{U} \rightarrow U$ under the functor

$$
\mathcal{C} \stackrel{(\tilde{U})^{*}}{\longrightarrow} \mathcal{C} / \tilde{U} \stackrel{\Pi_{p}}{\longrightarrow} \mathcal{C} / U \stackrel{\Sigma_{U}}{\longrightarrow} \mathcal{C} .
$$

Now to ask that $U$ is closed under dependent products is equally well to ask that $q$ is obtained as a pullback of $p$. We may similarly say what it means for $U$ to be closed under dependent sums, or for it to admit an interpretation of the identity types (essentially by assuming the existence of a well-behaved factorisation of the diagonal map $\tilde{U} \rightarrow \tilde{U} \times_{U} \tilde{U}$ in $\mathcal{C}$ ). These requirements are enough to ensure 
that the contextual category $\mathbb{C}(\mathcal{C}, p)$ admits interpretations of the $\Sigma$, $\Pi$, and Id type-constructors.

The particular instance of the above process that we have in mind is the following. Let $\mathcal{C}$ be the category of simplicial sets. Let $U$ denote the simplicial set whose $n$-simplices are Kan fibrations $f: X \rightarrow \Delta_{n}$, where $\Delta_{n}$ is the standard $n$-simplex and $f$ has fibers which are well-ordered and of cardinality $<\kappa$ (here $\kappa$ is a cardinal with sufficiently good closure properties; for instance, an inaccessible cardinal). Now there is a Kan fibration $p: \tilde{U} \rightarrow U$ with well-ordered fibers of cardinality of $<\kappa$ which is universal amongst such, in the sense that every other such Kan fibration is a pullback of it. A priori, this pullback is not necessarily unique; but this Kan fibration has a further special property, that of being univalent in a sense that we shall not make precise here - at low dimensions, it means that if two of its fibers are weakly equivalent, then they lie over points joined by a path in $U$, so that "each fiber appears at most once". This univalence ensures that the space of ways in which a map $q$ may appear as a pullback of $p$ is contractible; so that every small Kan fibration is a pullback of $p$ "uniquely up to homotopy".

It follows that $p$ is closed under all the type-theoretic constructors of the ambient category of simplicial sets; for example, the universal dependent family $q: \tilde{V} \rightarrow V$ is itself a Kan fibration with $\kappa$-small, well-ordered fibers, and hence appears as a pullback of the universal small Kan fibration, so that $p$ is closed under dependent products; and so on. Thus the contextual category $\mathbb{C}(\mathcal{C}, p)$ models all the constructors of Martin-Löf type theory. Furthermore, the fact that the Kan fibration $p$ is univalent allows us to prove that the corresponding contextual category validates the univalence axiom, as desired.

\section{A Coq tutorial}

Andrej Bauer

In this tutorial we show how to use the Coq proof assistant. We cover the basic usage of tactics, propositional and predicate calculus, and basic inductive definitions. Special attention is devoted to the inductive definition of propositional equality, as it plays a central role in the homotopy-theoretic interpretation of type theory. The accompanying material also shows a streamlined proof of Vladimir Voevodsky's result that the Univalence Axiom and Eta rule imply the principle of Function Extensionality. Our proof was developed during the workshop with Peter Lumsdaine, following a suggestion of Steve Awodey.

The materials used in the tutorial, as well as links to other resources about Coq and homotopy type theory, are available at http://math.andrej.com/. 
Mini-Workshop: The Homotopy Interpretation of Constructive Type Theory 11

\section{Introduction to Type Theory and its Semantics \\ THOMAS STREICHER}

From work of H. B. Curry it is well-known that simply typed $\lambda$-calculus can serve as a proof term assignment for the implicational fragment of intuitionistic logic. It is also well known that the semantics of simply typed $\lambda$-calculus is given by cartesian closed categories. Later W. Howard suggested to give a proof term assignment for full predicate logic via an extension of simply typed $\lambda$-calculus. This line of thought resulted at the end of the 1960s in Martin-Löf's Theory of Dependent Types. The first version assumed a type of all types containing itself as an element which was soon proved to be inconsistent by J.-Y. Girard. As a reaction to this inconsistency Martin-Löf developed his predicative type theory with a stratified hierarchy of (predicative) universes. About the same time J.-Y. Girard developed his impredicative system $F$ serving as a proof term assigment for second order logic. It took about fifteen more years till Martin-Löf's and Girard's work was synthesized by Th. Coquand and G. Huet in their Calculus of Constructions which a bit later was extended by a hierarchy of universes and inductive definition. This Extended Calculus of Constructions (ECC) is the underlying system of the Coq system, an interactive theorem prover used by a lot of computer scientists and mathematicians interested in actual formalization. In my talk I will describe how to model such type systems categorically and what adaptions are needed to model their intensional variants.

The most basic system is $\Pi$-calculus which differs from simply typed $\lambda$-calculus by allowing types to depend on variables of previously defined types. A (typing) context is an expression of the form $\Gamma \equiv x_{1}: A_{1}, \ldots, x_{n}: A_{n}$ where the $x_{i}$ are pairwise distinct and the free variables of $A_{i}$ are contained in the list $x_{1}, \ldots, x_{i-1}$. The rules for dependent product types are essentially those of the simply typed $\lambda$-calculus. The main difference is the $\Pi$-formation rule

$$
\frac{\Gamma \vdash A \quad \Gamma, x: A \vdash B}{\Gamma \vdash(\Pi x: A) B}
$$

where one gets $A \rightarrow B$ as the particular case where $B$ does not depend on $A$.

Contexts will be interpreted in a category $\mathcal{C}$ of contexts with a terminal object 1 corresponding to the empty context. Substitutions between contexts are interpreted as morphisms in $\mathcal{C}$. Families of types are interpreted as elements of a subclass $\mathcal{D}$ of $\operatorname{Mor}(\mathcal{C})$, so-called display maps. We write $a: A \longrightarrow I$ to indicate that $a \in \mathcal{D}$. Representing $I$-indexed families of objects in $\mathcal{C}$ as morphisms in $\mathcal{C}$ with codomain $I$ is inherited wisdom from geometry and logic. That families of types are stable under substitution is reflected by the assumption that $\mathcal{D}$ is stable under pullbacks along arbitrary morphisms in $\mathcal{C}$, i.e.

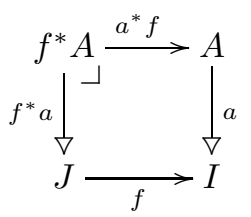


This assumption guarantees that $a$ induces a pullback functor $a^{*}: \mathcal{C} / I \rightarrow \mathcal{C} / A$. For interpreting dependent products we assume that for every $b: B \longrightarrow A$ the presheaf $\mathcal{C} / A\left(a^{*}(-), b\right)$ is representable by some $\Pi_{a} b: P \longrightarrow I$, i.e. there exists a morphism ev : $a^{*} \Pi_{a} b \rightarrow b$ such that for every $f: J \rightarrow I$ in $\mathcal{C}$ and $g: a^{*} f \rightarrow b$ in $\mathcal{C} / A$ there is a unique $\lambda(g): f \rightarrow \Pi_{a} b$ with ev $\circ a^{*} \lambda(g)=g$ as in

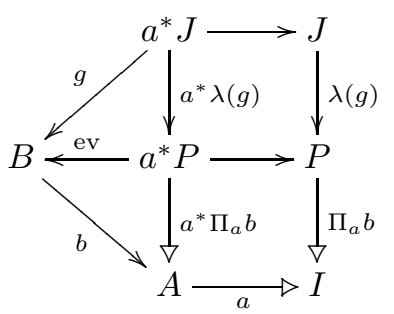

where all squares are pullbacks. Accordingly, as first observed by R. Seely every locally cartesian closed category $\mathcal{C}$ gives rise to an example when choosing $\mathcal{D}$ as $\mathcal{C}$. In order to interpret the equality rules for types we have to assume that pullbacks of display maps are chosen in a functorial way and that canonical pullback functors preserve $\Pi$ and ev on the nose. In case of the intensional variant of the $\Pi$-calculus we must not assume the $\lambda(g)$ to be unique w.r.t. their required property but only that there is a functorial choice of them. ${ }^{1}$

Dependent sum types are interpreted by composition of display maps and identity types as fibrewise diagonals. Of course, for this purpose $\mathcal{D}$ has to be closed under these operations. In the latter case this means that

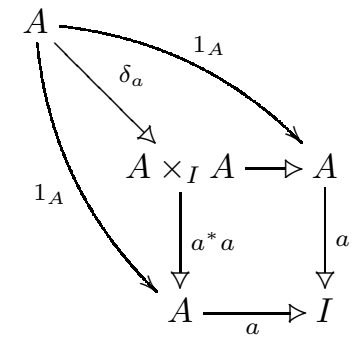

for every display map $a: A \longrightarrow I$. In order to interpret equality of types we have to assume that for $a: A \longrightarrow I$ and $b: B \longrightarrow A$ we have
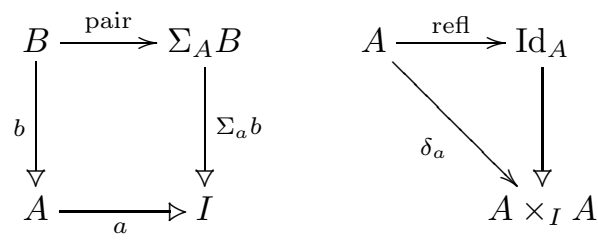

where pair and refl are isomorphisms and these choices are preserved by canonical pullbacks.

\footnotetext{
${ }^{1}$ In the simply typed case this amounts to a category $\mathcal{C}$ with finite products such that every presheaf $\mathcal{C}(-\times A, B)$ appears as retract of some representable presheaf $\mathcal{C}-, A \rightarrow B$.
} 
Mini-Workshop: The Homotopy Interpretation of Constructive Type Theory 13

Recall that from the Yoneda lemma it follows that a morphism $c_{A}: P_{A} \rightarrow A$ is an isomorphism iff for all $f: P_{A} \rightarrow C$ there exists a unique map $E(f): A \rightarrow C$ with $E(f) \circ c_{A}=f$. If we assume that from every object in $\mathcal{C}$ there is a finite sequence of display maps towards 1 (as common in models of type theory) the map $c_{A}$ is an isomorphism iff for all $c: C \longrightarrow A$ and $f: P_{A} \rightarrow C$ with $c \circ f=c_{A}$ there exists a unique section $E(f)$ of $c$ with $E(f) \circ c_{A}=f$. On the syntactical level in type theory this is expressed in terms of so-called elimination rules where $c_{A}$ is understood as a "constructor" and $E(f)$ as defined from $f$ by "pattern matching". In models of intensional type theory one does not require section $E(f)$ to be unique w.r.t. its required property but instead that this choice is stable under canonical pullbacks.

The most innovative idea of Martin-Löf Type Theory was that of a universe. Categorically, this is modeled by a display map $e: E \longrightarrow U$ in $\mathcal{D}$ such that the class $\mathcal{U}=\left\{f^{*} e \mid f: I \rightarrow U\right\}$ is closed under the respective operations corresponding to various type formation rules. We call a universe $\mathcal{U}$ impredicative iff for all $a: A \rightarrow I$ in $\mathcal{D}$ and $p: P \rightarrow A$ in $\mathcal{U}$ the map $\Pi_{a} p$ is in $\mathcal{U}$, too. This kind of universes is used for interpreting the impredicative type of propositions of ECC.

The most well known example is a topos $\mathcal{C}$ with $\mathcal{D}=\mathcal{C}$ and $\mathcal{U}$ the class of all monos. This is a so called proof irrelevant model in the sense that every map in $\mathcal{U}$ has at most one section. The most well known proof relevant models are categories $\operatorname{Asm}(\mathcal{A})$ of so-called assemblies over a partial combinatory algebra $\mathcal{A}$ as e.g. natural numbers with Kleene application. Here one takes for $\mathcal{D}$ all maps of $\operatorname{Asm}(\mathcal{A})$ and for $\mathcal{U}$ the class of families of so-called modest sets, i.e. assemblies where objects are uniquely determined by their realizers.

History and some references.

Display map semantics for type theory was introduced independently by various people in the mid 1980s, namely M. Hyland et.al. in Cambridge, F. Lamarche in Montreal and myself. Later on this was given a reformulation in terms of fibrations by Th. Ehrhard and B. Jacobs. This is nice from a conceptual point of view but its generality is not witnessed by any convincing examples. The most accessible account on the appropriate level of generality are A. Pitts' type categories as can be found in his survey article on Categorical Logic in the Handbook of Logic in Computer Science. In my old book Semantics of Type Theory one finds display map semantics and a "split" version of it in terms of J. Cartmell's contextual categories as favoured again in recent work of V. Voevodsky.

\section{Introduction to Homotopy Theory Michael Shulman}

Homotopy theory began, roughly, with the study of topological spaces up to deformation rather than homeomorphism. For instance, the plane is not homeomorphic to a point, but it can be "contracted" to a point via a continuous deformation. More precisely, a homotopy $f \sim g$ between two maps $f, g: X \rightarrow Y$ is a map $H: X \times[0,1] \rightarrow Y$ such that $H(-, 0)=f$ and $H(-, 1)=g$, and a homotopy 
equivalence is a map $f: X \rightarrow Y$ such that there exists $g: Y \rightarrow X$ and homotopies $g f \sim 1_{X}$ and $f g \sim 1_{Y}$.

A basic invariant of homotopy type is the set of path-components, $\pi_{0}(X)$. Another is the fundamental group $\pi_{1}(X, x)$ at $x \in X$ : the set of loops in $X$ based at $x$ modulo basepoint-preserving homotopy, with group structure induced by concatenation of paths. We put these together into the fundamental groupoid, whose objects are the points of $X$ and whose morphisms are homotopy classes of paths.

We can go on to define the higher homotopy groups $\pi_{n}(X, x)$ as the set of based maps $S^{n} \rightarrow X$ modulo homotopy. These are abelian for $n \geq 2$, by the "EckmannHilton argument", and we can put them all together into a fundamental $n$-groupoid or $\infty$-groupoid, modulo a suitable definition of such things.

We may ask whether homotopy groups completely determine a space up to homotopy. The answer is no, but we can define a nice class of spaces for which they do: $C W$ complexes, which are built by gluing together discs of various dimensions. Whitehead's theorem says that a map between CW complexes inducing an isomorphism on all homotopy groups (called a "weak homotopy equivalence") is actually a homotopy equivalence. In fact, the homotopy theory of CW complexes is naturally equivalent to that of their "fundamental $\infty$-groupoids."

Doing mathematics "up to homotopy" also requires homotopical versions of standard categorical constructions. Limits like equalizers and pullbacks are not homotopy invariant, but we can define "homotopy" versions of them which are. For instance, a point of the homotopy pullback $X \times{ }_{Z}^{h} Y$ consists of a point $x \in X$, a point $y \in Y$, and a path between their images in $Z$. Such "homotopy limits" are characterized, up to homotopy equivalence, by a homotopical universal property.

The homotopy limit is also the actual limit of a different diagram. For instance, the homotopy pullback of $f: A \rightarrow B$ is the ordinary pullback of $P f \rightarrow B$, where a point of $P f$ consists of a point $a \in A$ and a path in $B$ starting at $f(a)$. This map $P f \rightarrow B$ is a fibration, meaning that any homotopy $g \sim h$ in $B$ lifts to $P f$ once we specify a lifting of $g$. Moreover, the homotopy pullback of a fibration is homotopy equivalent to its actual pullback.

Finally, the homotopy lifting property means that any homotopy commutative square between fibrations can be rectified to a strictly commutative one. Thus fibrations have a property similar to $\mathrm{CW}$ complexes: all the morphisms between them that "should be there" on the homotopy level are actually there on the strict level. Taking this analogy further leads to Quillen model categories.

\section{Homotopical aspects of type theory Richard GaRner}

The objective of this talk was to draw together the threads spun by the preceding tutorials on dependent type theory and homotopy theory, by sketching the fundamental observation which links the two areas together. The starting point is the assignation sending a dependent type theory $\mathbb{T}$ to its classifying category $\mathcal{C}(\mathbb{T})$. 
Recall that this category has as objects, dependent contexts of variable declarations $\Delta=\left(x_{1}: A_{1}, x_{2}: A_{2}\left(x_{1}\right), \ldots, x_{n}: A_{n}\left(x_{1}, \ldots, x_{n-1}\right)\right)$ in the type theory $\mathbb{T}$, and as morphisms between them, dependent vectors of term assignations. For each type judgement $\Gamma \vdash A$ type of $\mathbb{T}$ there is a distinguished morphism $\pi_{A}$ of $\mathcal{C}(\mathbb{T})$ whose codomain is the context $\Gamma$ and whose domain is the context $\Gamma . A$ obtained by extending $\Gamma$ with $A$; it is given by projecting away the final component of the context. We refer to such morphisms as dependent projections and write $\mathcal{D}$ for the class of dependent projections in $\mathcal{C}(\mathbb{T})$. Observe that such a $\mathcal{D}$-map admits pullbacks along any morphism of $\mathcal{C}(\mathbb{T})$ - corresponding to type substitution in $\mathbb{T}$ - and the pullback is then itself a $\mathcal{D}$-map.

Consider now a type theory $\mathbb{T}$ which admits Martin-Löf's intensional identity types. Thus, for each type $A$ (let us consider only a closed type for simplicity), we have a type $x: A, y: A \vdash \operatorname{Id}_{A}(x, y)$ type; and this corresponds to having a $\mathcal{D}$-map $p: I_{A} \rightarrow A \times A$ in $\mathcal{C}(\mathbb{T})$. We have further a judgement $x: A \vdash \operatorname{r}(x)$ : $\operatorname{Id}_{A}(x, x)$ witnessing the reflexivity of identity; and this corresponds to a map $r: A \rightarrow I_{A}$ in $\mathcal{C}(\mathbb{T})$ whose composite with $p$ is the diagonal $A \rightarrow A \times A$. We have moreover the elimination rule for identity types, which states that whenever we have $x, y: A, p: \operatorname{ld}_{A}(x, y) \vdash C(x, y, p)$ type and $x: A \vdash d(x): C(x, x, \mathrm{r}(x))$, then we have a term $x, y: A, p: \operatorname{ld}_{A}(x, y) \vdash \mathrm{J}_{d}(x, y, p): C(x, y, p)$ satisfying $x: A \vdash J_{d}(x, x, \mathrm{r}(x))=d(x): C(x, x, \mathrm{r}(x))$. But this is equally well to say that whenever we are given a diagram

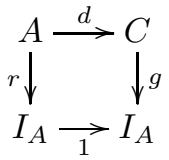

in $\mathcal{C}(\mathbb{T})$, with $g \in \mathcal{D}$, we can find a diagonal filler $j: I_{A} \rightarrow C$ making both squares commute. This is in turn to say that the map $r$ has the left lifting property with respect to any $\mathcal{D}$-map with codomain $I_{A}$. But since $\mathcal{D}$-maps are stable under pullback, this is further equivalent to the statement that $r$ has the left lifting property with respect to every $\mathcal{D}$-map. Writing $\mathcal{I}$ for the class of all maps with the left lifting property with respect to $\mathcal{D}$, we may therefore characterise $\mathbb{T}$ 's possession of identity types as the condition that every diagonal $A \rightarrow A \times A$ in $\mathcal{C}(\mathbb{T})$ should factor as the composite of an $\mathcal{I}$-map followed by a $\mathcal{D}$-map.

This is reminiscent of the definition of a weak factorisation system in the sense of Bousfield-Quillen: wherein one is presented with a category $\mathcal{C}$ equipped with two classes of maps $\mathcal{L}$ and $\mathcal{R}$, each closed under retracts in the arrow category, and such that every $\mathcal{L}$-map has the left lifting property with respect to every $\mathcal{R}$ map, and every map $f$ factors as $f=g h$ with $h \in \mathcal{L}$ and $g \in \mathcal{R}$. In particular, we might hope for two things: that whenever $\mathbb{T}$ is a type theory, $\mathcal{C}(\mathbb{T})$ supports a weak factorisation system, derived in some manner from the identity type structure; and conversely, that whenever a category $\mathcal{C}$ suitable for the modelling of dependent type theory supports a weak factorisation system of an appropriate kind, then it also supports the interpretation of Martin-Löf's identity types. The first of these was 
proven in [3], and the second in [1] - though in this direction, there are some subtle coherence issues to deal with, which are considered in detail in [2] and [4].

\section{REFERENCES}

[1] S. Awodey and M.A. Warren - Homotopy theoretic models of identity types, Mathematical Proceedings of the Cambridge Philosophical Society 146 (2009), no. 1, pp. 45-55.

[2] B. van den Berg and R. Garner - Topological and simplicial models of identity types. Accepted for publication in the ACM Transactions on Computational Logic (TOCL). Available as arXiv:1007.4638.

[3] N. Gambino and R. Garner - The identity type weak factorisation system, Theoretical Computer Science 409 (2008), no. 1, pp. 94-109.

[4] M.A. Warren - Homotopy theoretic aspects of constructive type theory, Ph.D. thesis, Carnegie Mellon University, 2008.

\section{Equality and dependent type theory}

\section{Thierry Coquand}

We give a purely axiomatic presentation of equality in type theory. Starting from a dependent type theory with dependent sum and products, basic types such as $N$ (type of natural numbers), $N_{0}$ (empty type) and $N_{1}$ (unit type), we axiomatize the equality type following the interpretation of types as spaces. We introduce a new type $\operatorname{Id}_{A} x_{0} x_{1}$ for $A$ type and $x_{0} x_{1}: A$ together with an element $1_{x}: \operatorname{Id}_{A} x x$ for $x: A$ and an operation $(\cdot): B\left(a_{0}\right) \rightarrow \operatorname{Id}_{A} a_{0} a_{1} \rightarrow B\left(a_{1}\right)$ if $B(x)$ is family of type over $A$, and the condition $\operatorname{Id}_{B(a)}\left(b \cdot 1_{a}\right) b$ for each $a: A$ and $b: B(a)$. If $A$ is a type, following Voevodsky, we define iscontr $A$ to be

$$
\Sigma a: A . \Pi x: A . \operatorname{ld}_{A} a x
$$

The next axiom states that the space of paths from a given point is contractible

$$
\text { iscontr }\left(\Sigma x: A . \operatorname{ld}_{A} \text { a } x\right)
$$

Modulo the other axioms, this last axiom is equivalent to the elimination rule given by Ch. Paulin: given $C(x, \alpha)$ for $x: A$ and $\alpha: \operatorname{ld}_{A} a x$ we have

$$
\text { elim : } C\left(a, 1_{a}\right) \rightarrow \Pi x: A . \Pi \alpha: \operatorname{Id}_{A} \text { a } x . C(x, \alpha)
$$

with the "computation" rule: $\operatorname{Id}_{C\left(a, 1_{a}\right)}\left(\operatorname{elim} c a 1_{a}\right)$ c for any $c: C\left(a, 1_{a}\right)$. Notice however that this rule is here expressed as a propositional equality and not as a new definitional equality added to type theory. Though we don't add a new definitional equality, but only new axioms, all the usual properties of equality seem to be derivable. For instance, any type has a groupoid structure, and any function defines a functor. Given a type $X$ we can define the loop space $\Omega(X, a)=\operatorname{Id}_{X}$ a a and it has a group structure. We can iterate this construction $\Omega_{n+1}(X, a)=$ $\Omega_{n}\left(\Omega(X, a), 1_{a}\right)$ and prove that $\Omega_{n}(X, a)$ is commutative for $n \geqslant 2$, which is a formal version of Čech, 1932, result on the commutativity of higher homotopy group. (More generally we can prove that if $X$ with a binary operation and an element $e: X$ which is both a left and right unit for this operation then the loop space $\Omega(X, e)$ is commutative.) We can then formulate the univalence 
axiom, and prove that it implies functional equality. Another consequence of the univalence axiom is that isomorphic structures are equal. For instance on the type $S=\Sigma X: U . X \times(X \rightarrow X)$ we have $\operatorname{Id}_{S}(X, a, f)(Y, b, g)$ iff the structures $(X, a, f)$ and $(Y, b, g)$ are isomorphic. This is joint work with Nils Anders Danielsson, who has checked all the proofs formally in an implementation of type theory.

The usual computation rule for equality type is obtained by defining the equality to be the least reflexive relation. This gives a computational justification of all but the last axiom (the univalence axiom). With this interpretation the equality $\operatorname{ld}_{B(a)}\left(b \cdot 1_{a}\right) b$ holds as a definitional equality. We conjecture that a computational interpretation of all the axioms is possible, defining the equality on a type by recursion on this type (extending the definition of Gandy 1956 for simple type theory). For this interpretation, we expect the equality $\operatorname{Id}_{B(a)}\left(b \cdot 1_{a}\right) b$ to hold only as a propositional equality and not as a definitional equality.

\section{The Univalence Axiom and Function Extensionality Nicola Gambino}

Vladimir Voevodsky has recently introduced a model of Martin-Löf's Constructive Type Theory which validates not only the standard rules for identity types, but also an additional principle, called the Univalence Axiom. The aim of this talk is to present the Univalence Axiom and to illustrate Voevodsky's proof that it implies the principle of Function Extensionality, which is the assertion that if two functions are pointwise propositionally equal, then they propositionally equal as elements of the appropriate function type.

The outline of the proof is as follows. First, one observes that the Univalence Axiom implies that if $f: X \rightarrow X^{\prime}$ is a weak equivalence, then for every type $Y$, the composition function $(-) \circ f:\left(X^{\prime} \rightarrow Y\right) \rightarrow(X \rightarrow Y)$ is again a weak equivalence. Secondly, using the fact that a homotopy equivalence is a weak equivalence, one shows that the homotopy diagonal $\delta_{Y}: Y \rightarrow \operatorname{ld}(Y)$ is a weak equivalence. Combining these two facts, we obtain that composition with $\delta_{Y}$,

$$
(-) \circ \delta_{Y}:(\operatorname{Id}(Y) \rightarrow Y) \rightarrow(Y \rightarrow Y),
$$

is a weak equivalence. This implies that the two projection functions

$$
\pi_{1}, \pi_{2}: \operatorname{ld}(Y) \rightarrow Y
$$

are propositionally equal. Indeed, $\pi_{1} \circ \delta_{Y}$ and $\pi_{2} \circ \delta_{Y}$ are propositionally equal, since they are both propositionally equal to the identity $1_{Y}: Y \rightarrow Y$. But composition with $\delta_{Y}$ is a weak equivalence and so $\pi_{1}$ and $\pi_{2}$ must be propositionally equal.

It is now straightforward to derive the principle of Function Extensionality. Indeed, suppose that we are given two functions $f_{1}, f_{2}: X \rightarrow Y$ and a proof $\phi: \Pi x: X . \mathrm{Id}_{Y}\left(f_{1} x, f_{2}\right)$ that they are pointwise propositionally equal. We can then define a function $f: X \rightarrow \operatorname{ld}(Y)$ such that $\pi_{1} \circ f$ is propositionally equal to $f_{1}$ and $\pi_{2} \circ f$ is propositionally equal to $f_{2}$ (using the $\eta$-rule). Since, as claimed above, $\pi_{1}$ is propositionally equal to $\pi_{2}$, we have that $\pi_{1} \circ f$ is propositionally equal to $\pi_{2} \circ f$, which implies that $f_{1}$ is propositionally equal to $f_{2}$, as required. 


\section{Logic-enriched type theory \\ Peter Aczel}

My talk presented the idea of a logic-enriched type theory and stated that there is a natural 'logical version', $\left[U A^{l} \Rightarrow F E^{l}\right]$, of Vladimir Voevodsky's result, proved in the Coq computer system, that there is a closed term of type $[U A \rightarrow F E]$. Here $U A$ is a closed type expressing Voevodsky's Univalence Axiom and $F E$ is a closed type expressing Function Extensionality.

To give more detail, let $\mathbb{U}$ be a type universe. The type $U A$ is

$$
(\Pi X, Y: \mathbb{U}) W e q(E X Y),
$$

where, for $f: A \rightarrow B, W e q(f)$ is a type that expresses that $f$ is a weak equivalence, and

$$
E:(\Pi X, Y: \mathbb{U})\left[I d_{\mathbb{U}}(X, Y) \rightarrow(\Sigma f: X \rightarrow Y) W e q(f)\right],
$$

is a canonical function defined using the elimination rule for $\mathbb{U}$ and a function $E_{0}:(\Pi X: \mathbb{U}) W e q\left(i d_{X}\right)$. Also $F E$ is the type

$$
\begin{array}{r}
(\Pi X, Y: \mathbb{U})\left(\Pi f_{1}, f_{2}: X \rightarrow Y\right) \\
{\left[\operatorname{ExtE}_{X \rightarrow Y}\left(f_{1}, f_{2}\right) \rightarrow I d_{X \rightarrow Y}\left(f_{1}, f_{2}\right)\right],}
\end{array}
$$

where $E x t E q_{X \rightarrow Y}\left(f_{1}, f_{2}\right)$ is $(\Pi x: X) I d_{Y}\left(f_{1} x, f_{2} x\right)$. The logical versions of $U A$ and $F E$ are the following, where we use $\sim_{X}$ for the propositional equality relation on the type $X$ and let $\operatorname{Tr}(X)$ be the proposition $(\exists x: X)\left[x \sim_{X} x\right]$. The proposition $U A^{l}$ is

$$
(\forall X, Y: \mathbb{U}) \operatorname{Tr}(W e q(E X Y))
$$

and $F E^{l}$ is the proposition

$$
\begin{aligned}
& (\forall X, Y: \mathbb{U})\left(\forall f_{1}, f_{2}: X \rightarrow Y\right) \\
& \quad\left[\operatorname{Tr}\left(\operatorname{ExtEq_{X}\rightarrow Y}\left(f_{1}, f_{2}\right)\right) \Rightarrow\left(f_{1} \sim_{X \rightarrow Y} f_{2}\right)\right]
\end{aligned}
$$

A Logic-enriched type theory is a dependently sorted predicate logic whose dependent sorts are the dependent types of a type theory. I focused on a logicenrichment $T T^{l}$ of a version, $T T$ of Martin-Löf's intensional type theory, the logic being intuitionistic predicate logic with equality combined with induction principles for the inductive types of the type theory. It is possible to add to $T T^{l}$ a logical version $A C^{l}$ of the type-theoretic Axiom of Choice. Given a type $A$, a family of types, $B(x)$ for $x: A$, and a predicate, $R(x, y)$ for $x: A, y: B(x), A C^{l}$ is the proposition

$$
\begin{aligned}
(\forall x: A)(\exists y: B(x)) R(x, y) & \\
\quad \Rightarrow & (\exists f:(\Pi x: A) B(x))(\forall x: A) R(x, f x) .
\end{aligned}
$$

Some metatheorems concerning the relationship between $T T^{l}+A C^{l}$ and $T T$ were presented. These expressed that $T T^{l}+A C^{l}$ characterises the logic obtained in the [propositions = types] treatment of logic. A key feature of the proof of $\left[U A^{l} \Rightarrow F E^{l}\right]$ is that it takes place in $T T^{l}$ without any use of $A C^{l}$. The advantage of this is that $T T^{l}$ has a much richer variety of interpretations than $T T^{l}+A C^{l}$. 


\section{Remarks on homotopical logic}

ANDRÉ JOYAL

Formal logic, like any formal system, can be analysed with the tools of abstract algebra and category theory. The algebraic analysis of logic began with George Boole in the 19th century and was pursued by Tarski in the 20th century with the notion of cylindric algebra, and by Heyting with the notion of Heyting algebra. The categorical analysis of logic, which began with the work of Lambek and Lawvere, has transformed deeply our conception of algebraic logic by adding a kind of conceptual layer between the syntax and the semantics. More explicitly, to any formal logical system is associated a term model which is a category equipped with certain structure. The properties of the category of terms can be described quite independently of the actual syntax of the formal system. Ideally, every formal system of logic should exhibit three layers: a conceptual layer which specifies a certain class $\mathbf{C}$ of categories and functors, a semantic layer which specifies natural examples of categories in $\mathbf{C}$ (the semantic domains) and a formal layer which specifies a language and a deduction system for constructing algebraically the categories in $\mathbf{C}$. The layers are not independent of each other and each clarifies the others. But the conceptual layer has the central role as a kind of middle-man:

$$
\text { Formal layer } \leftarrow \text { Conceptual layer } \rightarrow \text { Semantic layer }
$$

For example, the conceptual layer of classical predicate logic can be taken to be the class of Boolean pretopoi, its semantic layer to be the category of sets, and its formal layer to be the syntax and deduction rules of predicate calculus. Of course, this description has many variants since the layers may be chosen differently. For example, all Boolean Grothendieck topoi could be included in the semantics of classical predicate logic.

The conceptual layer of (pure) dependent type theory consists of categories $\mathcal{C}$ equipped with a class $\mathcal{D}$ of display maps closed under base changes (and possibly under composition) (see the talk of Thomas Streicher). We may suppose in addition that $\mathcal{C}$ has a terminal object 1 and that the unique map $A \rightarrow 1$ is a display map for every object $A$. The conceptual layer of type theory with products $\Pi$ can be taken to be a category $\mathcal{C}$ with a class of display maps $\mathcal{D}$ closed under internal products in the following sense: the product $f_{*}(X)$ of a display map $X \rightarrow A$ along a display map $f: A \rightarrow B$ exists and is a display map $f_{*}(X) \rightarrow B$. If $\mathcal{D}=\mathcal{C}$, this means that the category $\mathcal{C}$ is locally cartesian closed. On the other hand, the conceptual layer of intensional type theory was missing until quite recently. A breakthrough was the groupoid interpretation of Hofmann and Streicher [5]. We now know that intensional type theory has a natural semantics in a Quillen model category $\mathcal{E}$, thanks to the work of Awodey and Warren [2]. The category $\mathcal{C}$ is the full subcategory of fibrant objects of $\mathcal{E}$ and the display maps are the fibrations; the equality type $\operatorname{ld}_{A} \rightarrow A \times A$ is the path space as constructed by Quillen. In order to interpret the internal products $\Pi$ we suppose that the category $\mathcal{E}$ is locally cartesian closed and that the model structure right proper. This is true for example of the classical model structure on the category of simplicial sets, and 
more generally of the model structure (introduced by myself) on the category of simplicial objects of any Grothendieck topos

It is obvious that homotopy theory has had a strong influence on the development of many branches of mathematics during the last twenty years. Today, we have $A^{1}$-homotopy theory (Morel, Voevodsky), a theory of higher stacks (Hirschowitz, Simpson), homotopical algebraic geometry (Toen and Vezzosi), derived algebraic geometry (Deligne, Drinfel'd, Kontsevich, Kapranov, Ciocan-Fontanine, Toen, Vezzosi, Lurie) and topological algebraic geometry (Lurie, Toen, Vezzosi). Many new objects and ideas were introduced in these developments. A new structural foundation based on $(\infty, 1)$-categories, rather than on ordinary categories, was put in place. Roughly speaking, an $(\infty, 1)$-category is a category enriched over the category of homotopy types. The main examples are simplicial categories (Dwyer and Kan), Segal categories (Hirschowitz and Simpson), complete Segal spaces (Rezk) and quasi-categories (Bordman and Vogt / Joyal). In his book "Higher topos theory" Lurie has demonstrated that essentially all the categorical constructions of SGA4 can be extended from categories to quasi-categories, hence also to all $(\infty, 1)$-categories [7]. There is a similar trend in algebra, starting with the $A_{\infty}$-algebras of Stasheff. Boardman and Vogt have given a general mechanism by which one can create homotopy coherent versions of any classical algebraic structure.

$$
\text { categories } \rightarrow \infty \text {-categories, algebras } \rightarrow \infty \text {-algebras, topoi } \rightarrow \infty \text {-topoi }
$$

It seems natural to ask the question

$$
\operatorname{logic} \rightarrow \infty \text {-logic? }
$$

I conjecture that, in the presence of the function extensionality axiom of Voevodsky, intensional type theory is a formal system for working in a locally cartesian closed $(\infty, 1)$-category. More precisely, let me describe the notions involved in this conjecture. There is a notion of quasi-category with finite products $[6,7]$. If $X$ and $Y$ are quasi-categories with finite products, then a map $f: X \rightarrow Y$ preserves finite products if it takes each terminal object in $X$ to a terminal object of $Y$ and if the canonical morphism $f(x \times y) \rightarrow f(x) \times f(y)$ is invertible for every pair of objects $x, y \in X$. A quasi-category with finite products $X$ is cartesian closed if the map $x \times(-): X \rightarrow X$ has a right adjoint $[x,-]: X \rightarrow X$ for every object $x$ in $X$. If $X$ and $Y$ are cartesian closed quasi-categories, then a map $f: X \rightarrow Y$ is cartesian closed if it preserves finite products and the canonical morphism $f[x, y] \rightarrow[f(x), f(y)]$ is invertible for every pair of objects $x, y \in X$. We shall denote the category of cartesian closed quasi-categories by $C C Q$.

Recall that the category of quasi-categories QCat is enriched over itself, since it is cartesian closed. It is thus enriched over the category of Kan complexes if we put $\operatorname{Hom}[X, Y]=J[X, Y]$, where $J(Z)$ is the maximal sub Kan complex of a quasi-category $Z$. If $X$ and $Y$ are cartesian closed quasi-categories, then the full simplicial subset of $\operatorname{Hom}[X, Y]$ spanned by the cartesian closed maps $X \rightarrow Y$ is a Kan complex $C C[X, Y]$. This defines an enrichment of the category $C C Q$ over the category of Kan complexes. 
Mini-Workshop: The Homotopy Interpretation of Constructive Type Theory 21

If $A$ is a simplicial set, we shall say that a cartesian closed quasi-category $F(A)$ equipped with a map $i: A \rightarrow F(A)$ is freely generated by $A$ if the restriction map

$$
i^{*}: C C[F(A), X] \rightarrow \operatorname{Hom}[A, X]
$$

is a homotopy equivalence for every cartesian closed quasi-category $X$.

A quasi-category $X$ has finite limits if it has a terminal object and the quasicategory $X / x$ has finite products for every object $x \in X$. A quasi-category with finite limits $X$ is locally cartesian closed if the quasi-category $X / x$ is cartesian closed for every object $x \in X$. If $X$ and $Y$ are locally cartesian closed quasicategories, then a map $f: X \rightarrow Y$ is a locally cartesian closed if it preserves terminal objects and the induced map $X / x \rightarrow Y / f(x)$ is cartesian closed for every object $x \in X$. There is a notion of locally cartesian closed quasi-category freely generated by a simplicial set.

Recall that a homotopical category is a pair $(\mathcal{C}, W)$ where $\mathcal{C}$ is a category and $W \subseteq \mathcal{C}$ is a class of morphisms called weak equivalences [3]. There is then a quasi-category $L(\mathcal{C}, W)$ equipped with a map $i: \mathcal{C} \rightarrow L(\mathcal{C}, W)$ which inverts universally every morphism in $W$. The quasi-category $L(\mathcal{C}, W)$ is the Dwyer-Kan localisation of $\mathcal{C}$ with respect to $W$. It can be constructed by first attaching to (the nerve of) $\mathcal{C}$ a left and a right inverse to every arrow in $W$, and then by taking a fibrant replacement of the resulting simplicial set (in the model structure for quasi-categories) [6].

If $G$ is a graph, let us denote by $M L T T(G)$ the type system obtained by adjoining to Martin-Löf intensional type theory a type $T(a)$ for each vertex $a \in G$ and a term $f$ of type $T(a) \rightarrow T(b)$ for each arrow $f: a \rightarrow b$ in $G$. Let us denote by $C T(G)$ the term category associated to $M L T T(G)$ as in [4]. Let us denote by $W$ the set of display maps in $C T(G)$ which are weak equivalences in the sense of Voevodsky.

Conjecture The quasi-category $L(C T(G), W)$ is locally cartesian closed and it is freely generated by $G$ as a one dimensional simplicial set.

\section{ConClusion}

Homotopical logic could be defined as a (new) branch of logic which studies structured homotopy types rather than structured sets. Sets are homotopy types of $h$-level 1 (Voevodsky). The goal of Univalent Foundations is to replace sets by homotopy types in the foundation of mathematics. I hope the project is successful. But not all mathematical logic is concerned with foundational questions and the same should be true of homotopical logic. A large part of model theory studies groups, fields, graphs, etc. Homotopical model theory may study ring spaces, spectra, ring spectra, etc. But a field is defined more by its methods than by its objects. It is not yet clear that homotopical model theory can be different from homotopy theory itself.

In order to accommodate homotopical logic, I would like to introduce a fourth layer in my schematic description of formal logic. The conceptual layer may be defined by a class of structured quasi-categories rather than by a class of structured 
categories. Every quasi-category is the Dwyer-Kan localisation of a homotopical category $(\mathcal{C}, W)$ (in a non-unique way). In homotopical logic, the purpose of the formal system is to generate $(\mathcal{C}, W)$ algebraically. We may say that $(\mathcal{C}, W)$ belongs to the algebraic layer.

Formal layer $\leftarrow$ Algebraic layer $\leftarrow$ Conceptual layer $\rightarrow$ Semantic layer.

\section{REFERENCES}

[1] S. Awodey, Type theory and homotopy theory (2010, available at http://www. andrew. cmu.edu/user/awodey/)

[2] S. Awodey, M. Warren, Homotopy theoretic models of identity types Mathematical Proceedings of the Cambridge Philosophical Society 146 (2009) no.1, pp. 45-55.

[3] W. Dwyer, P. S. Hirschhorn, D. M. Kan, J.H. Smith, Homotopy Limit functors and Homotopical Categories, AMS Mathematical Surveys and Monographs, Volume 113 (2004).

[4] N. Gambino, R. Garner, The identity type weak factorisation system, Theoretical Computer Science 409 (2008), no. 1, pp. 94-109.

[5] M. Hofmann, T. Streicher, The groupoid interpretation of type theory Twenty-five years of constructive type theory Proceedings of a congress, Venice, Italy, October 19-21, 1995. Oxford: Clarendon Press. Oxf. Logic Guides. 36, 83-111 (1998).

[6] A. Joyal, The theory of quasi-categories, in preparation.

[7] J. Lurie, Higher Topos Theory, (2008) available at http://arxiv.org/abs/math/0608040.

\section{A combinatorial realizability model of 1-truncated type theory} Michael A. WARREN

(joint work with Pieter Hofstra)

Let $\mathbb{T}_{1}$ be the theory obtained by adding to intensional Martin-Löf type theory the 1-truncation rule

$$
\frac{p: \operatorname{Id}_{\mathbf{I d}_{A}(a, b)}(f, g)}{f=g: \operatorname{Id}_{A}(a, b)}
$$

which states the the usual reflection rule holds when applied to types which are themselves already identity types. It follows from [2] that each closed type $A$ in this theory has an associated groupoid $|A|$ with objects the closed terms of type $A$ and with hom-sets given by $|A|(a, b)$ the set of closed terms of type $\operatorname{Id}_{A}(a, b)$. When $G$ is a (directed) graph we obtain the 1-truncated theory $\mathbb{T}_{1}[G]$ with $G$ adjoined by adding to $\mathbb{T}_{1}$ a new basic type $\ulcorner G\urcorner$ together with the axioms

- $\ulcorner a\urcorner:\ulcorner G\urcorner$ for each vertex $a$ of $G$.

- $\ulcorner f\urcorner: \operatorname{Id}\ulcorner G\urcorner(\ulcorner a\urcorner,\ulcorner b\urcorner)$ for each edge $f$ from $a$ to $b$.

Henceforth, Gödel brackets $\ulcorner$.$\urcorner are omitted, as it is always clear from context$ when we are speaking of vertices and edges and when we are speaking of their type-theoretic representatives. Sending a graph $G$ to the underlying graph of $|G|$ gives a monad $T$ on the category Graph of graphs and, following [1], the algebras are called 1-truncated Martin-Löf complexes. Understanding the behavior of these algebras is a first step in the project initiated in ibid of modeling homotopy types using the higher-categorical structures arising from type theory. 
Mini-Workshop: The Homotopy Interpretation of Constructive Type Theory 23

In this connection, we observe that the monad $T$ gives rise to new vertices. In particular, $T(G)$ will in general have infinitely many vertices even when the graph $G$ is finite. For example, if $f$ is a loop in the graph $G$ on a vertex $a$ and $b$ is any vertex of $G$, the elimination rule for identity types gives

$$
\frac{x, y: G, z: \operatorname{Id}_{G}(x, y) \vdash G \quad x: G \vdash b: G}{\mathrm{~J}([x: G] b, a, a, f): G}
$$

where the first two hypotheses are simply obtained by weakening. One would like to know that such "duplicate" or "doppelgänger" terms do nothing homotopically harmful. E.g., $T(G)$ should have the same connected components as $G$. Similarly, from a logical point of view one would like to know that there are no non-standard terms of natural number type in $\mathbb{T}_{1}[G]$ in the sense that we would like to prove that for each term $t$ of natural number type there exists a numeral $\mathrm{n}$ and a term of type $\operatorname{Id}(t, \mathrm{n})$. (Whether there exist non-standard terms of natural number type in the presence of Voevodsky's univalence axiom is a related question and we expect that the techniques mentioned in this abstract can be modified to yield a proof of Conjecture 1 from [5].) We will now describe the model construction (appearing in full in [3]) which allows us to answer these and related questions. This construction is inspired by the logical relations method due to Tait [4].

We begin by describing the general interpretation of closed terms and types:

- A closed type $A$ will be interpreted as a functor $\llbracket A \rrbracket:|A| \rightarrow$ Set. Intuitively, $\llbracket A \rrbracket(a)$ is the set of realizers of $a$ and we write $\alpha \Vdash a: A$ to indicate that $\alpha$ is an element of this set.

- A closed term $a: A$ will be interpreted as an element of $\llbracket A \rrbracket(a)$. (Explicitly, as a global section of $\llbracket x: A \rrbracket=\int \llbracket A \rrbracket$ which projects to $a$.)

The description for open terms proceeds inductively based on the lengths of contexts. For the sake of brevity we describe the simple case of an open type $x: A \vdash B(x)$ depending only on a single free variable. In this case, we have a pseudofunctor $|x: A \vdash B|:|A| \rightarrow \mathbf{G p d}$ which sends an object $a$ of $|A|$ to the groupoid $|B(a)|$ and acts on arrows by the usual "reindexing" term. We write $|x: A, y: B(x)|$ for the groupoid $\int|x: A \vdash B(x)|$ obtained by the Grothendieck construction. Given $\llbracket A \rrbracket$ we define $\|x: A, y: B(x)\|$ to be the pullback of the projection $|x: A, y: B| \rightarrow|x: A|$ along the projection $\llbracket x: A \rrbracket \rightarrow|x: A|$. I.e., $\|x: A, y: B(x)\|$ has as objects tuples $(a, \alpha, b)$ where $a: A, \alpha \Vdash a$ and $b: B(a)$. Then we have the following:

- $\llbracket x: A \vdash B(x) \rrbracket$ is a functor $\|\Gamma, y: B\| \rightarrow$ Set.

- An open term $x: A \vdash b(x): B(x)$ is interpreted as a section of the projection $\llbracket x: A, y: B(x) \rrbracket \rightarrow \llbracket x: A \rrbracket$ such that $\llbracket b(x) \rrbracket(a, \alpha)$ is of the form $(a, \alpha, b(a), \beta)$.

In terms of realizers, this means that to define the interpretation of an open type $x: A \vdash B(x)$ is precisely to give a notion of realizer

$$
\beta \Vdash_{\alpha} b: B(a)
$$


for $(a, \alpha, b)$ an object of $\|x: A, y: B(x)\|$ such that there is a functorial action

$$
\beta \cdot(f, g) \Vdash_{\alpha^{\prime}} b^{\prime}: B\left(a^{\prime}\right)
$$

on realizers, for arrows $(f, g):(a, \alpha, b) \rightarrow\left(a^{\prime}, \alpha^{\prime}, b^{\prime}\right)$ in $\|x: A, y: B(x)\|$. Similarly, to give the interpretation of an open term $x: A \vdash b(x): B(x)$ is precisely to give an assignment

$$
b[\alpha] \Vdash_{\alpha} b(a): B(a)
$$

for each $\alpha \Vdash a: A$, which is required to satisfy a simple coherence condition. This completes the description of the general setting of the model and we may now describe the interpretation of the various terms and types of the theory explicitly. We begin by describing the interpretation of the basic type $G$.

Consider the groupoid model (see $[2]$ ) of $\mathbb{T}_{1}[G]$ obtained by interpreting the type $G$ as the free groupoid $\mathcal{F}(G)$ on $G$. Under this interpretation a closed term $a: G$ will be interpreted as an object of $\mathcal{F}(G)$ (a vertex of the graph $G$ ). This interpretation restricts to a functor $|G| \rightarrow \mathcal{F}(G)$ and composing it with the canonical functor $\mathcal{F}(G) \rightarrow|G|$ gives an endofunctor ${ }^{-}$on $|G|$ which associates to each closed term $a$ of type $G$ a basic term $\bar{a}$ of type $G$ called the closure of $a$. Similarly, given a closed term $f: \operatorname{Id}_{G}(a, b)$ we obtain $\bar{f}: \operatorname{Id}_{G}(\bar{a}, \bar{b})$, where this term $\bar{f}$ is a composite of basic terms and inverses of basic terms. We say that a map $f: a \rightarrow b$ in $|G|$ is dense if $\bar{f}$ is definitionally equal to the reflexivity term $\operatorname{r}(\bar{a})$ in $\mathbb{T}_{1}[G]$. Then, we define,

$$
\alpha \Vdash a: G \quad \text { iff } \quad \alpha \text { is a dense map } a \rightarrow \bar{a} .
$$

The functorial action on realizers is easily given by defining $\alpha \cdot f \Vdash b$, for $f: a \rightarrow b$, to be the composite $\bar{f} \circ \alpha \circ f^{-1}$. To take another example, given $A$ together with $\llbracket A \rrbracket, \alpha \Vdash a: A, \beta \Vdash b: A$ and a closed term $f: \operatorname{ld}_{A}(a, b)$ we define

$$
\xi \Vdash_{\alpha, \beta} f: \operatorname{ld}_{A}(a, b) \quad \text { iff } \quad \alpha \cdot f=\beta \text { and } \xi=\emptyset .
$$

I.e., realizers are proofs that $\alpha \cdot f=\beta$. Realizers at dependent product type are given by sections, realizers at dependent sum type are given by pairs and realizers at natural number type are given exactly as for $G$.

We then obtain the following consequences:

Theorem 1. The interpretation described above is sound and complete with respect to the theory $\mathbb{T}_{1}[G]$.

Corollary 2. The natural numbers in $\mathbb{T}_{1}[G]$ are standard in the sense that if a is any term of natural number type, then there exists a numeral $\mathrm{n}$ and a propositional equality $a \rightarrow \mathrm{n}$.

Corollary 3. The canonical map $\mathcal{F}(G) \rightarrow|G|$ is a categorical equivalence.

Proof. It suffices to construct a natural isomorphism $\eta: 1_{|G|} \rightarrow^{-}$. We take as the component $\eta_{a}: a \rightarrow \bar{a}$ the realizer from $\llbracket a \rrbracket$. This determines a natural since, given $f: a \rightarrow b$, the realizer of $f$ from $\llbracket f \rrbracket$ is a proof that $\bar{f} \circ \eta_{a} \circ f^{-1}=\eta_{b}$.

Corollary 4. $T(G)$ has the same connected components as $G$. 
Mini-Workshop: The Homotopy Interpretation of Constructive Type Theory 25

\section{REFERENCES}

[1] S. Awodey and P. Hofstra and M. A. Warren, Martin-Löf Complexes, (2009), submitted.

[2] M. Hofmann and T. Streicher, The groupoid interpretation of type theory, (1998) in TwentyFive Years of Constructive Type Theory G. Sambin and J. Smith eds., 1998, 83-111.

[3] P. Hofstra and M. A. Warren, Combinatorial realizability models of type theory, in preparation.

[4] W. W. Tait, Intensional interpretations of functionals of finite type I, Journal of Symbolic Logic, 32 (1967), 198-212.

[5] V. Voevodsky, Univalent foundations project, (2010), a modified version of an NSF grant application.

\section{Homotopy-theoretic models of type theory \\ BENNO VAN DEN BERG \\ (joint work with Richard Garner)}

Martin-Löf Type Theory was developed by Per Martin-Löf in 1973 [9] with the aim of providing a rigorous foundation for predicative, constructive mathematics. As was stressed later by Martin-Löf (see [10]) the theory can also be regarded as a functional programming language, due to the proofs-as-programs paradigm. On this paradigm, a constructive proof for a certain statement can be regarded as a program which computes a witness.

The theory went through several phases before it reached its final mature form. One point which remained unsettled for a long time were the rules for the identity types. Martin-Löf's idea is that "types" are the constructive analogue of sets and are always built up inductively. However, in some of the older formulations of type theory the rules for the identity types did not conform to this philosophy (see, for example, [11], which in all other respects contains the mature system). For every type $A$ and elements $a, b \in A$ there is a new type $\operatorname{Id}(A, a, b)$, to be thought of as the set of proofs saying that the terms $a$ and $b$ denote equal elements of $A$. The older "extensional" formulation of the rules for the identity type essentially say that the type $\operatorname{Id}(A, a, b)$ contains at most one element and that this type is inhabited precisely when the terms $a$ and $b$ have the same normal form. This was later rejected, essentially for two reasons. First of all, this formulation did not fit in the general philosophy, as it prevented the type $\operatorname{Id}(A, a, b)$ from being inductively generated. Secondly, it made type checking undecidable. The intensional formulation of the identity types avoid both problems: they are formulated in a way which conforms to the general philosophy and they keep type checking decidable.

There is a price to pay, however, for the intensional identity types are much harder to work with and much harder to understand. But in recent years unexpected connections to ideas from higher-dimensional category theory and algebraic topology have started to shed light on the combinatorics of the intensional identity types (besides the references mentioned below, see also [1, 12, 13].

For this the seminal paper has been the one by Hofmann and Streicher [6] where they show that the rules for the intensional identity type give every type the structure of a groupoid (a category in which every arrow is invertible). Moreover, they 
gave a model of type theory in which types $A$ are interpreted as groupoids, elements $a, b$ as objects, and the identity type $\operatorname{Id}(A, a, b)$ as the set (discrete groupoid) of arrows from $a$ to $b$. Using this model they managed to refute the uniqueness of identity proofs in intensional type theory. So whereas in the extensional type theory it is built in that there can be only one proof of the equality of $a$ and $b$, this is not provable in intensional type theory: indeed, what Hofmann and Streicher show is that in the model based on groupoids there are generally more. But, as they remark, it is still the case that proofs of equalities have to be equal in the model (as $\operatorname{Id}(A, a, b)$ is interpreted as a discrete groupoid), but there is no reason to expect this to be provable in the type theory. Indeed, in general, one would expect $\operatorname{Id}(A, a, b)$ to have a groupoidal structure as well, and the same applies to the iterated identity type $\operatorname{Id}(\operatorname{Id}(A, a, b), p, q)$, etcetera. So the resulting structure on each type $A$ should not be just groupoidal, or even 2-groupoidal, but that of a (weak) $\omega$-groupoid.

As a matter of fact, in work with Richard Garner (see [3]), we prove that this is correct: the resulting structure is that of a weak $\omega$-groupoid. ${ }^{1}$ A striking aspect of our proof is that it works for rather abstract reasons which have little to do with the syntax of type theory (the structure one needs for the proof to work can be formulated entirely in terms of what are called weak factorisation systems; to see how these arise from type theory, consult [5]). This means it can be applied in other settings as well. Indeed, it can be used to show that the fundamental weak $\omega$-groupoid of a space ${ }^{2}$ is indeed a weak $\omega$-groupoid in the sense of BataninLeinster. That we have a uniform proof that both type theory and homotopy theory give rise to weak $\omega$-groupoids shows that the analogy goes much deeper than one might initially suspect.

In subsequent work with Richard Garner [4], we have taken the natural next step, which is to use ideas from homotopy theory to build models for the intensional identity types. It turns out that it all works as one would hope, so that the category of topological spaces can be used to model the identity types, when interpreting the identity types as path spaces. In fact, we have a simple notion of a "path object category" and show how the intensional identity types can be interpreted in these path object categories. We also show that not only does the category of topological spaces have the structure of such a path object category, but also the category of simplicial sets can be endowed with such a structure.

\section{REFERENCES}

[1] S. Awodey and M.A. Warren - Homotopy theoretic models of identity types, Mathematical Proceedings of the Cambridge Philosophical Society 146 (2009), no. 1, pp. 45-55.

\footnotetext{
${ }^{1}$ As it happens, there are many different notions of weak $\omega$-groupoid, but the one that was most suited to our purposes was that of Batanin [2], as modified by Leinster [7]. The same result was independently proved by Peter Lumsdaine [8].

${ }^{2}$ Start with a topological space $X$ and associate to it its set of points, paths between those points, homotopies between those paths, homotopies between homotopies, etc. The resulting structure is called the fundamental weak $\omega$-groupoid of the space.
} 
[2] M.A. Batanin - Monoidal globular categories as a natural environment for the theory of weak n-categories, Advances in Mathematics 136 (1998), no. 1, pp. 39-103.

[3] B. van den Berg and R. Garner - Types are weak $\omega$-groupoids, Proceedings of the London Mathematical Society (3) 102 (2011), pp. 370-394.

[4] B. van den Berg and R. Garner - Topological and simplicial models of identity types. Accepted for publication in the ACM Transactions on Computational Logic (TOCL). Available as arXiv:1007.4638.

[5] N. Gambino and R. Garner - The identity type weak factorisation system, Theoretical Computer Science 409 (2008), no. 1, pp. 94-109.

[6] M. Hofmann and T. Streicher - The groupoid interpretation of type theory, in: Twentyfive years of constructive type theory (Venice, 1995), Oxford Logic Guides 36, pp. 83-111. Oxford University Press, New York, 1998.

[7] T. Leinster - Higher operads, higher categories, London Mathematical Society Lecture Note Series 298, Cambridge University Press, Cambridge, 2004.

[8] P.L. Lumsdaine - Weak $\omega$-categories from intensional type theory, Logical Methods in Computer Science 6, no. 3, 3:24, 19 pages, 2010.

[9] P. Martin-Löf - An intuitionistic theory of types: predicative part, in: Logic Colloquium 73 (Bristol, 1973), Studies in Logic and the Foundations of Mathematics 80, pp. 73-118. North-Holland, Amsterdam, 1975.

[10] P. Martin-Löf - Constructive mathematics and computer programming, Philosophical Transactions of the Royal Society of London (Series A) 312 (1984), no. 1522, pp. 501-518.

[11] P. Martin-Löf - Intuitionistic type theory, Studies in Proof Theory 1, Bibliopolis, Naples, 1984.

[12] V. Voevodsky - The univalent foundations project. Manuscript available from the author's homepage.

[13] M.A. Warren - Homotopy theoretic aspects of constructive type theory, Ph.D. thesis, Carnegie Mellon University, 2008.

\section{Open problems session}

A short session was convened during the workshop to discuss how the programme of homotopy type theory should be taken forward and in which directions further research should be directed. A broad range of ideas was discussed, and these were crystallised into the following short list of questions.

- What are the potential benefits of the Univalence Axiom (UA) for the practice of (higher) category theory?

- In Coq, given a term $t$ : nat constructed with the use of UA, is there is a terminating algorithm which constructs a term $t_{0}$ : nat which does not use UA and a term (whose construction may well use UA) in $\operatorname{Id}_{\text {nat }}\left(t, t_{0}\right)$ ?

- Does UA have models in other categories (e.g. 1-topoi) not equivalent to the standard one?

- Can we extend type theory with a homotopically meaningful notion of colimit/cofibration?

- What is $S_{n}$ (in Coq)?

- What is a simplicial type?

- Can the notion of proposition be implemented in Coq in a way which does not commit to the interpretation given in the Calculus of Constructions? 
- Does there exist a univalent (weakly universal Kan) fibration in the category of simplicial objects in an arbitrary topos?

Reporter: Richard Garner 